Western University

Scholarship@Western

Brain and Mind Institute Researchers'

Publications

Brain and Mind Institute

4-29-2016

Progression from Vegetative to Minimally Conscious State Is Associated with Changes in Brain Neural Response to Passive Tasks: A Longitudinal Single-Case Functional MRI Study

\author{
Francesco Tomaiuolo \\ Unità Gravi Cerebrolesioni Acquisite \\ Luca Cecchetti \\ Unità Gravi Cerebrolesioni Acquisite \\ Raechelle M. Gibson \\ The University of Western Ontario \\ Fiammetta Logi \\ Unità Gravi Cerebrolesioni Acquisite \\ Adrian M. Owen \\ The University of Western Ontario, uwocerc@uwo.ca
}

See next page for additional authors

Follow this and additional works at: https://ir.lib.uwo.ca/brainpub

Citation of this paper:

Tomaiuolo, Francesco; Cecchetti, Luca; Gibson, Raechelle M.; Logi, Fiammetta; Owen, Adrian M.; Malasoma, Franco; Cozza, Sabino; Pietrini, Pietro; and Ricciardi, Emiliano, "Progression from Vegetative to Minimally Conscious State Is Associated with Changes in Brain Neural Response to Passive Tasks: A Longitudinal Single-Case Functional MRI Study" (2016). Brain and Mind Institute Researchers' Publications. 983.

https://ir.lib.uwo.ca/brainpub/983 


\section{Authors}

Francesco Tomaiuolo, Luca Cecchetti, Raechelle M. Gibson, Fiammetta Logi, Adrian M. Owen, Franco Malasoma, Sabino Cozza, Pietro Pietrini, and Emiliano Ricciardi 


\title{
Progression from Vegetative to Minimally Conscious State Is Associated with Changes in Brain Neural Response to Passive Tasks: A Longitudinal Single-Case Functional MRI Study
}

\author{
Francesco Tomaiuolo, ${ }^{1,2,3}$ Luca Cecchetti, ${ }^{1,2,3}$ Raechelle M. Gibson, ${ }^{4}$ Fiammetta Logi, ${ }^{1}$ Adrian M. Owen, \\ Franco Malasoma, ${ }^{5}$ Sabino Cozza, ${ }^{5}$ Pietro Pietrini, ${ }^{2,3,6}$ AND Emiliano Ricciardi ${ }^{3}$ \\ ${ }^{1}$ Unità Gravi Cerebrolesioni Acquisite, Auxilium Vitae Volterra, Pisa, Italy \\ ${ }^{2}$ Clinical Psychology Branch, Pisa University Hospital, Pisa, Italy \\ ${ }^{3}$ Laboratory of Clinical Biochemistry and Molecular Biology, Department of Surgical, Medical and Molecular Pathology and Critical Care, \\ University of Pisa, Pisa, Italy \\ ${ }^{4}$ The Brain and Mind Institute, Department of Psychology, University of Western Ontario, London, N6A 5B7, Canada \\ ${ }^{5}$ U.O. Radiologia, ASL5 Volterra, Pisa, Italy \\ ${ }^{6}$ IMT School for Advanced Studies Lucca, Lucca, Italy
}

(Received November 2, 2015; Final Revision April 19, 2016; AcCePted May 2, 2016)

\begin{abstract}
Objectives: Functional magnetic resonance imaging (fMRI) may be adopted as a complementary tool for bedside observation in the disorders of consciousness (DOC). However, the diagnostic value of this technique is still debated because of the lack of accuracy in determining levels of consciousness within a single patient. Recently, Giacino and colleagues (2014) hypothesized that a longitudinal fMRI evaluation may provide a more informative assessment in the detection of residual awareness. The aim of this study was to measure the correspondence between clinically defined level of awareness and neural responses within a single DOC patient. Methods: We used a follow-up fMRI design in combination with a passive speech-processing task. Patient's consciousness was measured through time by using the Coma Recovery Scale. Results: The patient progressed from a vegetative state (VS) to a minimally conscious state (MCS). Patient's task-related neural responses mirrored the clinical change from a VS to an MCS. Specifically, while in an MCS, but not a VS, the patient showed a selective recruitment of the left angular gyrus when he listened to a native speech narrative, as compared to the reverse presentation of the same stimulus. Furthermore, the patient showed an increased response in the language-related brain network and a greater deactivation in the default mode network following his progression to an MCS. Conclusions: Our findings indicate that longitudinal assessment of brain responses to passive stimuli can contribute to the definition of the clinical status in individual patients with DOC and represents an adequate counterpart of the bedside assessment during the diagnostic decision-making process. (JINS, 2016, 22, 1-11)
\end{abstract}

Keywords: Disorders of consciousness, fMRI, Vegetative state, Minimally conscious state, Language processing, Longitudinal design

\section{INTRODUCTION}

Acquired brain injury can result in clinical conditions characterized by a complete (vegetative state, VS) or partial (minimally conscious state, MCS) loss of awareness. While VS patients do not show any evidence of a sustained, reproducible, purposeful, or voluntary behavioral response to visual, auditory, tactile, or noxious stimuli (Multi-Society Task Force on PVS, 1994), MCS individuals exhibit

Correspondence and reprint requests to: Luca Cecchetti, Via Roma, 67 - Building 43, 56100 Pisa, Italy. E-mail: cecchetti.luca@gmail.com inconsistent but reproducible signs of awareness, including the ability to follow simple commands, even though they remain unable to communicate interactively (Giacino et al., 2002). Along with brain death and coma, these clinical conditions are referred to as disorders of consciousness (DOC) (Bernat, 2006). The diagnosis of the DOC currently relies on the patient's clinical history and the bedside observation of the patient's behavioral responses to stimulations (Laureys, Owen, \& Schiff, 2004). Despite rigorous clinical assessment, the evaluation of residual awareness in individual patients is often problematic, and misdiagnosis rates are estimated to be as high as 43\% (Schnakers et al., 2009). 
Over the past decade, non-invasive neuroimaging methodologies have been used to assess residual awareness in patients with DOC (Fernandez-Espejo \& Owen, 2013). For example, to assess whether or not a patient can follow spoken commands in the absence of overt movement, one may ask the patient to engage in mental imagery. Using functional magnetic resonance imaging (fMRI), the patient's engagement in the mental imagery task can be quantified on the basis of the voluntary modulation of their brain activity.

Remarkably, one patient who fulfilled the clinical criteria of the VS exhibited a spatial and temporal pattern of brain activity that was nearly indistinguishable from that of fully conscious, healthy volunteers (Owen et al., 2006). Furthermore, one patient diagnosed as in an MCS was able to answer autobiographic questions using the same approach (Monti et al., 2010). Unfortunately, given that many patients with DOC have limited cognitive resources and fluctuating levels of attention and arousal, these so-called active tasks are not suitable for all patients in this special population (Monti, Pickard, \& Owen, 2013; Naci \& Owen, 2013).

To yield higher sensitivity in evaluating patients with DOC and limited cognitive resources (Giacino \& Smart, 2007), bedside examination of consciousness has been integrated with measures of neural activity elicited during passive stimulation tasks. For instance, passive paradigms include evaluating brain responses to simple sensory stimulation (Bekinschtein et al., 2004; Boly et al., 2008; Coleman et al., 2007; Di et al., 2007; Schiff et al., 2005) or assessing spontaneous brain activity and metabolism during rest (Laureys, Lemaire, Maquet, Phillips, \& Franck, 1999; Stender et al., 2014). Using a passive hierarchical speechcomprehension task, neural responses in language processing regions demonstrated a strong (although not unequivocal at the single patient level) overlap with the clinical assessment of awareness (Coleman et al., 2007).

Additionally, the functional integrity of the default mode network (DMN) at rest predicted residual awareness across the consciousness continuum, from brain death to coma and locked-in status (Boly et al., 2009; Demertzi et al., 2014; Hannawi, Lindquist, Caffo, Sair, \& Stevens, 2015; Vanhaudenhuyse et al., 2010). Despite the accumulating evidence that passive neuroimaging approaches can complement and even improve the assessment of the DOC, the diagnostic and prognostic value of these techniques is still questioned, and clinical practice remains largely unchanged.

One way to determine the real clinical efficacy of passive neuroimaging-based assessments is to conduct a longitudinal evaluation. By integrating neural responses during passive stimulation and clinical assessments at different time points in patients with prolonged DOC, a longitudinal evaluation could characterize the "neural signature" of residual awareness over time (Giacino, Fins, Laureys, \& Schiff, 2014). Importantly, as compared to cross-sectional designs, a longitudinal evaluation carried out within the single patient does not necessarily rely on group averaging; thus, it can be influenced less by the potential confounding effect introduced by between groups (e.g., VS . MCS) heterogeneous distribution of etiological, demographic, and medical factors, which also importantly influence prognosis of DOC (Bernat, 2006).

Although preliminary findings (Bekinschtein et al., 2005; Laureys et al., 1999) have shown a potential role for this approach to support clinical diagnosis within a single DOC patient, a combined description of language-related and default mode brain activity during recovery of consciousness is still lacking. To this end, here we present a longitudinal fMRI evaluation in combination with a passive auditory task to characterize neural responses in one patient who progressed from a VS to an MCS. Specifically, the patient's brain responses within language-related networks and the DMN were investigated over time while he passively listened to a meaningful short narrative played both forward and backward (using a paradigm previously validated in patients with DOC; Schiff et al., 2005). Since this speech perception task relies on both coding early aspects of language (Dehaene-Lambertz, Dehaene, \& Hertz-Pannier, 2002) and sustained attention during listening (Caplan \& Dapretto, 2001), we hypothesized that clinical progression toward consciousness would be associated with both selective responses in hierarchically higher language-related areas and parallel modulations in the DMN activity.

\section{METHODS}

\section{Patient Clinical History}

A 23-year-old, right-handed male was admitted to the intensive care unit in a comatose state following a severe head trauma sustained in a motor vehicle accident. Upon admission, the patient's score on the Glasgow Coma Scale (Teasdale \& Jennett, 1974) was 3. Whereas a first computed tomography examination did not reveal any noticeable pathological evidence, a second computed tomography scan 5 days later revealed a bilateral frontal hygroma. A subsequent brain MRI confirmed the bilateral hygroma and uncovered a diffuse axonal injury involving mainly the anterior frontal and temporal white matter, as well as the isthmus and splenium of the corpus callosum. In addition, the volume of the lateral ventricles was increased, and signs of cortical atrophy were evident in the bilateral frontal and right anterior temporal regions. There was no evidence of brainstem damage.

After 30 days, the patient's vital functions were stable, and he was transferred to our intensive rehabilitation unit. Here, the behavioral assessment of patient's awareness was conducted according to the revised version of the JFK Coma Recovery Scale (CRS-R; Kalmar \& Giacino, 2005) and his most relevant progressions toward consciousness are summarized in Table 1. Until 5 days before the first fMRI scan (approximately 3 months after admission), the patient was assessed daily by a multidisciplinary team, including an expert neuropsychologist, a neurologist, and a physiatrist. Pharmacological therapy comprised enteral baclofen, 
Table 1. Patient's most relevant advances toward consciousness as measured using the CRS-R scale

\begin{tabular}{|c|c|c|c|c|c|}
\hline Time since admission & 1 Month & 3 Months & 5 Months & 19 Months & 24 Months \\
\hline Diagnosis & VS & VS & MCS & MCS & EMCS \\
\hline \multicolumn{6}{|l|}{ Auditory Function Scale } \\
\hline 4 - Consistent Movement to Command* & & & & - & • \\
\hline 3 - Reproducible Movement to Command* & & & • & & \\
\hline 2 - Localization to Sound & & - & & & \\
\hline 1 - Auditory Startle & • & & & & \\
\hline \multicolumn{6}{|l|}{0 - None } \\
\hline \multicolumn{6}{|l|}{ Visual Function Scale } \\
\hline 5 - Object Recognition* & & & & & • \\
\hline 4 - Object Localization: Reaching* & & & & • & \\
\hline 3 - Visual Pursuit* & & & - & & \\
\hline \multicolumn{6}{|l|}{2 - Fixation* } \\
\hline 1 - Visual Startle & • & - & & & \\
\hline \multicolumn{6}{|l|}{0 - None } \\
\hline \multicolumn{6}{|l|}{ Motor Function Scale } \\
\hline 6 - Functional Object Use** & & & & & • \\
\hline 5 - Automatic Motor Response* & & & & - & \\
\hline \multicolumn{6}{|l|}{4 - Object Manipulation* } \\
\hline 3 - Localization to Noxious Stimulation* & & & • & & \\
\hline \multicolumn{6}{|l|}{2 - Flexion Withdrawal } \\
\hline 1 - Abnormal Posturing & • & • & & & \\
\hline \multicolumn{6}{|l|}{0 - None/Flaccid } \\
\hline \multicolumn{6}{|l|}{ Oromotor/Verbal Function Scale } \\
\hline \multicolumn{6}{|l|}{3 - Intelligible Verbalization* } \\
\hline 2 - Vocalization/Oral Movement & & & & $\bullet$ & $\bullet$ \\
\hline 1 - Oral Reflexive Movement & • & - & - & & \\
\hline \multicolumn{6}{|l|}{0 - None } \\
\hline \multicolumn{6}{|l|}{ Communication Scale } \\
\hline 2 - Functional: Accurate** & & & & & • \\
\hline 1 - Non-Functional: Intentional* & & & $\bullet$ & $\bullet$ & \\
\hline $0-$ None & - & - & & & \\
\hline \multicolumn{6}{|l|}{ Arousal Scale } \\
\hline 3 - Attention & & & & • & • \\
\hline 2 - Eye Opening w/o Stimulation & • & • & - & & \\
\hline \multicolumn{6}{|l|}{1 - Eye Opening with Stimulation } \\
\hline 0 - Unarousable & & & & & \\
\hline Total score & 6 & 7 & 13 & 19 & 22 \\
\hline
\end{tabular}

Note. Dashed lines represent the times of fMRI acquisitions. Total score cutoff for MCS is 8 .

*Denotes MCS.

***Denotes emergence from MCS

clonidine, diazepam, and propranolol. Throughout this period, the patient's behavior was consistent with a diagnosis of a VS: the patient was able to open his eyes spontaneously and had preserved, although inconsistent, reflexive behavior (visual and auditory startle). Automatic responses to noxious stimuli were evident, as well as oral reflexive movements. The patient did not exhibit overt motor responses to verbal command, visual fixation, or tracking of either auditory or visual stimuli. Importantly, the patient also did not demonstrate any behavior that could be regarded as "voluntary."

Approximately 2 months after the first fMRI scan, the patient's clinical status changed to an MCS. This clinical status change occurred because the patient became able to execute simple commands, such as visual fixation, systematically. The patient remained in an MCS for 1 year; approximately at the end of this 1-year period, he underwent a second fMRI scan (14 months since admission) following a routine clinical brain scan. At the time of acquisition, the patient reliably produced motor acts as a response to simple verbal command and was taking levetiracetam and sertraline.

Finally, 19 months after sustaining his brain injury, the patient was discharged from the hospital. The patient was assessed behaviorally 5 months after his discharge. At that time, the patient could not walk or stand, and he produced only basic vocalizations (i.e., no words). However, upon request, the patient was able to compute simple calculations and to interact and play with his mother (i.e., hit a balloon with his hand). According to his mother's report, the patient 
began to produce this behavior a few weeks after his discharge from the hospital. Together, this evidence suggested that the patient had emerged from an MCS to a clinical diagnosis of severe disability.

\section{Stimuli and Experimental Paradigm}

Linguistic stimuli were recorded in a quiet, anechoic room, through a professional USB microphone connected to a computer running Audacity 2.0.3 (http://audacity.source forge.net/). The stimuli consisted of (1) a 27-s story depicting neutral scenery with elements of high imageability (see Appendix), read by a female Italian speaker who was unfamiliar to the patient (forward speech condition, $\mathrm{Fw}$ ), and (2) a backward version of the same story obtained by reversing the speech file while maintaining sound pressure and pitch constant (backward speech condition, Bw). Both narratives were presented to the patient through MRcompatible headphones. Before the functional scan, the volume of the stimuli was adjusted by one of the experimenters to ensure a clear perception of the stimuli during the acquisition. Using a block-design paradigm, both conditions were alternated with $27 \mathrm{~s}$ of silence (rest condition) in each fMRI session. A total of six "stimulus-rest" repetitions were collected for both the Fw and Bw conditions during two separate fMRI runs.

The study was conducted under a protocol approved by the Ethical Committee of the Pisa University Hospital. After the study procedures and risks involved had been explained, a written informed consent was obtained from the patient's mother before each of the two acquisitions. Indeed, since there is no evidence that the use of fMRI with passive stimulation paradigms may provide a proof of patient's awareness or language comprehension, we were careful to avoid any kind of expectation in the patient's mother.

At the time of consent, we clearly informed her that: (1) no current diagnostic or prognostic information were expected from the fMRI acquisition and, therefore, there was no direct benefit to her son's clinical care; (2) the functional MRI experiment would be carried out at the time of the clinically scheduled routine structural MRI examinations; (3) the fMRI study would not involve any additional discomfort or risk for the patient, as compared to those associated with the standard clinical MRI examination; and (4) the fMRI study would require to prolong the MRI scanning session of approximately $12 \mathrm{~min}$.

Furthermore, we explained to the patient's mother that the additional fMRI scans were performed as a part of research aimed at exploring the neural underpinnings of consciousness. Importantly, as mentioned above, the two functional MRI acquisitions were performed concomitantly to the MRI clinical scans aimed at monitoring the evolution of the bilateral hygroma, brain atrophy, and at excluding the normal pressure hydrocephalus. The patient's mother gave her written informed consent before each of the two acquisitions and retained the right to withdraw from the study at any time with no explanation required. Finally, the fMRI scan exam was obtained at absolutely no cost for the patient's family.

\section{MR Acquisition}

Functional and structural brain images were acquired using a 1.5 Tesla (T) Siemens Symphony Magnetom MR system. The entire session had an approximate duration of $30 \mathrm{~min}$. The patient was carefully positioned in the scanner, MRcompatible headphones were provided, and cushions were used to minimize the patient's head movements. Throughout the functional scan, the experimenters ensured that the patient kept his eyes open by means of a head-coil mounted mirror.

For each of the two fMRI sessions, a gradient echo-echo planar imaging sequence [repetition time $(\mathrm{TR})=2.7 \mathrm{~s}$; echo time $(\mathrm{TE})=50 \mathrm{~ms}$; flip angle $(\mathrm{FA})=90^{\circ} ; 30$ interleaved axial slices; voxel size $3 \times 3 \times 3.75 \mathrm{~mm} ; 120$ volumes; 2 runs] together with a block-design paradigm were used to measure blood oxygenation level dependent (BOLD) changes during the passive speech perception task. A T2-weighted turbo spin echo sequence $\left(\mathrm{TR}=4.2 \mathrm{~s} ; \mathrm{TE}=109 \mathrm{~ms} ; \mathrm{FA}=90^{\circ} / 150^{\circ}\right.$; 33 axial slices; voxel size $0.6 \times 0.6 \times 3.75 \mathrm{~mm}$; number of excitations $=2$ ) was also acquired for clinical purposes and to define the anatomical locations of the task-related neural activations.

\section{Data Analyses}

The fMRI data were processed using FSL (Jenkinson, Beckmann, Behrens, Woolrich, \& Smith, 2012), and AFNI (Cox, 1996) in the following order: (1) optimized brain extraction (Lutkenhoff et al., 2014); (2) spatial alignment to the anatomical scan (FSL-FLIRT); (3) correction for slice time acquisition (3dTshift) and head motion (3dvolreg); (4) spatial smoothing using a Gaussian kernel of full-width half-maximum $=8 \mathrm{~mm}$ (3dmerge), and (5) mean scaling normalization. Afterward, brain responses to each condition (Fw and $\mathrm{Bw}$ ) were independently modeled using a block response function. The brain activity was evaluated in a GLM (3dDeconvolve) with nuisance variables represented by patient head movements (rotations and translations on $\mathrm{x}, \mathrm{y}$, and $\mathrm{z}$ axes). Maps of statistical significance ( $t$ values) for patient's brain responses were then obtained for the following contrasts: $\mathrm{Fw}>\mathrm{Bw}, \mathrm{Bw}>\mathrm{Fw},(\mathrm{Fw}+\mathrm{Bw})>$ Rest, Rest $>$ $(\mathrm{Fw}+\mathrm{Bw})$, using a $p<.05$ Bonferroni-corrected threshold.

To compare brain activity directly throughout the two stages of the patient's clinical status change (i.e., the first scan with his diagnosis as in a VS versus the second scan with his diagnosis as in an MCS) and to avoid circularity errors induced by selecting and testing using the same dataset, the BOLD signal was extracted for each fMRI session from meta-analytically defined regions of interest (ROIs; Figure 2B). These regions of interest corresponded to the language-related network, the DMN, and the visual motion perception network (as a task-unrelated control). To this end, we used the Neurosynth repository (Yarkoni, Poldrack, 
Table 2. Task-related brain activity at the time of first (VS Diagnosis) and second (MCS Diagnosis) fMRI examination

\begin{tabular}{|c|c|c|c|c|c|}
\hline \multicolumn{6}{|c|}{ VS diagnosis } \\
\hline Contrast & Region & $\mathrm{x}$ & $\mathrm{y}$ & $\mathrm{z}$ & T-Score \\
\hline \multirow[t]{2}{*}{$\mathrm{Fw}+\mathrm{Bw}>$ Rest } & Left superior temporal gyrus & -60 & -20 & 10 & 18.02 \\
\hline & Right superior temporal gyrus & 66 & -18 & 2 & 13.47 \\
\hline \multicolumn{6}{|c|}{ MCS diagnosis } \\
\hline Contrast & Region & $\mathrm{x}$ & $\mathrm{y}$ & $\mathrm{z}$ & T-Score \\
\hline \multirow[t]{3}{*}{$\mathrm{Fw}+\mathrm{Bw}>$ Rest } & Left superior temporal gyrus & -58 & -24 & 6 & 23.59 \\
\hline & Right superior temporal gyrus & 64 & -4 & -2 & 15.28 \\
\hline & Left inferior frontal gyrus & -46 & 8 & 16 & 8.62 \\
\hline \multirow[t]{5}{*}{ Rest $>\mathrm{Fw}+\mathrm{Bw}$} & Right precuneus & 14 & -60 & 24 & 10.05 \\
\hline & Right angular gyrus & 56 & -58 & 22 & 8.45 \\
\hline & Left anterior middle frontal & -34 & 32 & 10 & 8.34 \\
\hline & Left superior parietal lobule & -40 & -58 & 54 & 7.64 \\
\hline & Right anterior middle frontal & 24 & 66 & -2 & 7.41 \\
\hline $\mathrm{Fw}>\mathrm{Bw}$ & Left angular gyrus & -38 & -76 & 32 & 10.48 \\
\hline
\end{tabular}

Nichols, Van Essen, \& Waager, 2011) and topic-related reverse inference maps (Poldrack et al., 2012).

Specifically, for the language-processing network, our approach identified the following regions: the bilateral inferior frontal gyrus, the superior and middle temporal gyri, the left angular gyrus, the middle occipital gyrus, the fusiform gyrus, and the precentral gyrus (http://neurosynth. org/analyses/topics/v3-topics-50/4 - 636 studies, keywords e.g., sentences, comprehension, language). On the other hand, the meta-analytically defined DMN included: the anterior and posterior cingulate cortex, the bilateral temporo-parietal junction, the superior and middle frontal gyri, the middle temporal gyrus, and the parahippocampal gyrus (http://neurosynth.org/analyses/topics/v3-topics-50/01130 studies, keywords e.g., resting, default mode, intrinsic).

Finally, the task-unrelated control network (areas responding to visual motion perception) entailed the following regions: the bilateral hMT (posterior middle temporal gyrus); the fusiform and occipital gyri, and the superior parietal lobule (http://neurosynth.org/analyses/topics/v3topics-50/20 - 662 studies, keywords e.g., motion, visual, perception). These ROIs were computed in the standard space coordinates system; thus, the statistical maps for the patient were transformed spatially to match the MNI152 template using an affine registration (FSL-FLIRT). For each MR session (VS and MCS diagnosis) and each of the six $\mathrm{Fw}$ and six Bw stimulation blocks, average $t$ values were extracted independently from the three meta-analytically defined networks. Finally, the effect of the clinical progression toward consciousness was tested using a two-tailed paired $t$ test $(p<.05)$.

In addition, to evaluate the lateralization of the patient's brain activity both when he was in a VS and after he transitioned to an MCS, first, for each acquisition (i.e., first and second fMRI scan), we identified the coordinates for peak response of the $(\mathrm{Fw}+\mathrm{Bw})>$ Rest contrast within the left and right hemisphere. Afterward, similarly to what was done to test the effect of clinical progression toward consciousness, we extracted the average $t$ values of the six $\mathrm{Fw}$ and six $\mathrm{Bw}$ stimulation blocks from these coordinates, both when the patient met the criteria for a VS diagnosis and when he was in an MCS. Therefore, two independent two-tailed paired $t$ tests $(p<.05)$ were applied to determine significant differences between the right and left hemisphere response during VS and MCS.

\section{RESULTS}

\section{Task-Related Brain Responses}

The task-related brain responses are summarized in Table 2. When the patient was diagnosed as in a VS (the first fMRI scan), he showed a significant bilateral activation of the superior temporal cortex when both the forward and backward speech were presented, as compared to the silent rest period (Figure 1A; Fw $+\mathrm{Bw}>$ Rest). However, no brain regions were recruited differentially for the presentation of the forward speech as compared to the backward speech ( $\mathrm{Fw}$ $>\mathrm{Bw})$ or for the opposite contrast (Bw $>\mathrm{Fw})$.

When the patient had progressed to an MCS (the second fMRI scan), he generated significant neural activations in the bilateral superior temporal cortex and in the left inferior frontal cortex during the presentation of both the forward and the backward speech, as compared to the silent rest period (Figure 1B; $\mathrm{Fw}+\mathrm{Bw}>$ Rest). Importantly, the patient exhibited a significant activation in the left angular gyrus when presented with the forward speech as compared to the backward speech (Figure 2A; Fw > Bw). Using the same statistical threshold, no brain regions were recruited differentially for the opposite contrast ( $\mathrm{Bw}>\mathrm{Fw})$.

No regions were deactivated significantly in response to both types of speech stimuli when the patient was in a VS 
(a)

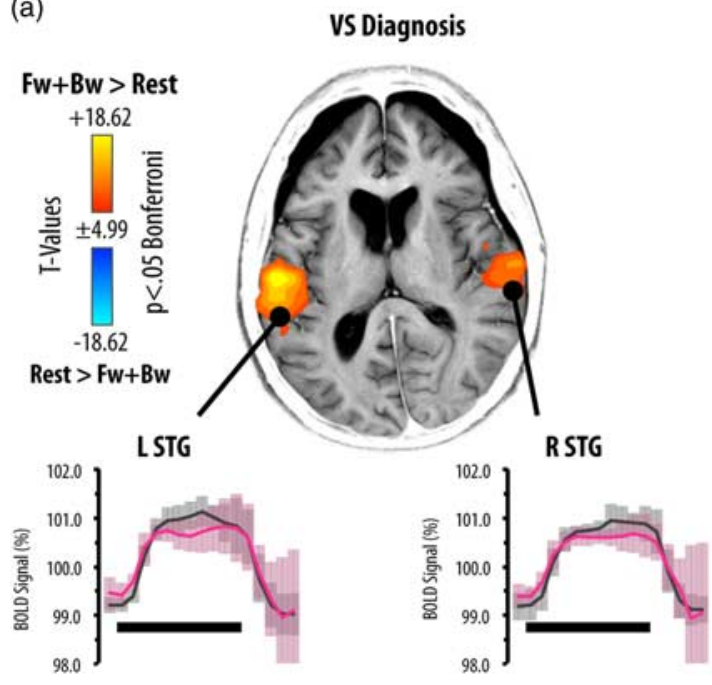

(b)

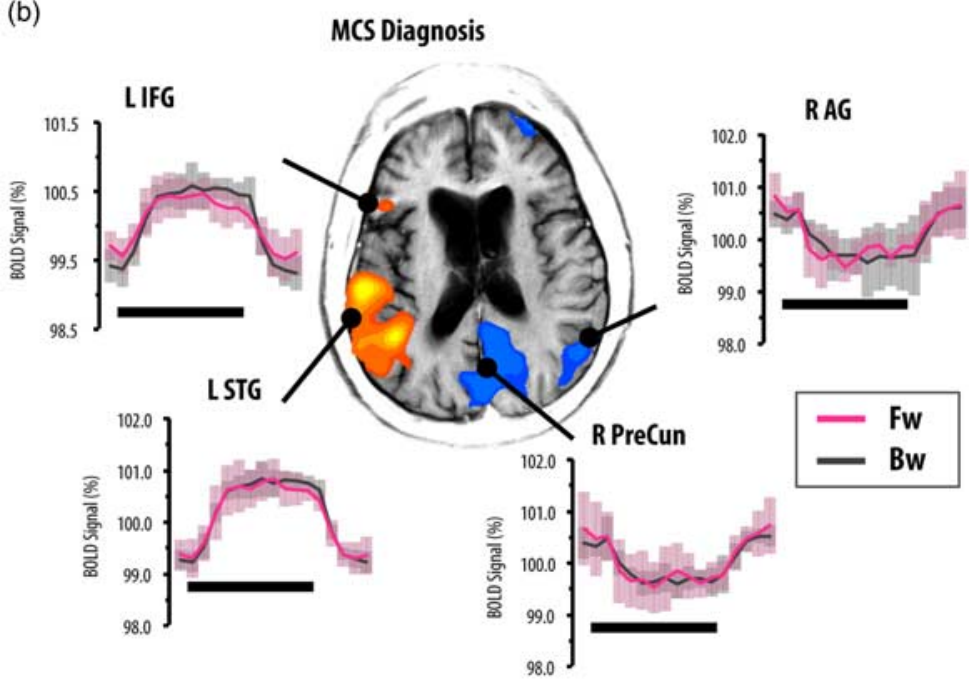

Fig. 1. This figure shows significant ( $p<.05$ Bonferroni corrected) activations (red-yellow clusters) related to both forward and backward speech conditions as compared to silent rest $(\mathrm{Fw}+\mathrm{Bw}>\mathrm{Rest}$ ), as well as deactivations (blue-cyan clusters) revealed by the opposite contrast (Rest $>\mathrm{Fw}+\mathrm{Bw}$ ). For each region, the average BOLD time-course of forward (fuchsia) and backward (dark grey) stimuli is shown, as well as the associated error (i.e., standard deviation; shaded areas). The thick black line located at the bottom of each plot represents the stimulus onset and duration (i.e., $27 \mathrm{~s}$ ). In the VS period (panel A) speech-related activations were limited to the bilateral superior temporal gyrus (L STG and R STG) and no brain regions were significantly deactivated. After the patient progressed to MCS, he additionally recruited the left inferior frontal gyrus (L IFG) and significantly deactivated important regions within the DMN, such as the right angular gyrus (R AG) and precuneus (R PreCun).

(Rest $>\mathrm{Fw}+\mathrm{Bw}$ ). Conversely, the right angular gyrus, the right precuneus, the left superior parietal lobule, and the bilateral anterior middle frontal gyrus showed significant deactivations when the patient was in an MCS and presented with both the forward and backward speech conditions (Figure 1B; Rest > Fw $+\mathrm{Bw}$ ).

\section{Changes in Brain Responses of Meta-analytically Defined ROIs Throughout Clinical Progression from a VS to an MCS}

The longitudinal assessment of the patient's brain responses as he progressed from a VS to an MCS resulted in a BOLD signal increment for the whole language-related network ( $p=.049$; Figure 2B, orange) and a greater deactivation within DMN brain regions ( $p=.022$; Figure 2B, purple). Neural activity for task-unrelated control brain areas associated with visual motion perception did not differ throughout his clinical progression from a VS to an MCS $(p=.454$; Figure $2 \mathrm{~B}$, green). Of note, these results were not driven by confounds related to head motion, since the average values for the two acquisitions did not differ significantly (VS versus MCS; two-tailed paired $t$ test; $p=.133$ )

\section{Lateralization of Peak Response}

Interestingly, while the patient was in a VS, the magnitude of BOLD activity extracted from the left hemisphere did not differ significantly $(p=.090)$ from the brain response recorded within the right hemisphere [left superior temporal gyrus $(-60,-20,+10)$; average $t$ values $=4.96 \pm 1.88$ and right superior temporal gyrus $(+66,-18,+2)$; average $t$ values $=4.05 \pm 1.08$, respectively]. On the contrary, while the patient was in an MCS, the hemodynamic activity measured within the left hemisphere was statistically higher $(p=.00072)$ compared to that one of the right hemisphere (left superior temporal gyrus $[-58,-24,+6]$; average $t$ values $=7.74 \pm 2.33$ and right superior temporal gyrus $[+64,-4,-2]$; average $t$ values $=5.23 \pm 2.07$, respectively).

\section{DISCUSSION}

In this work, we present the longitudinal fMRI investigation of an individual patient with a DOC whose clinical status progressed from a VS to an MCS. Our report explores the consistency between changes in the patient's task-related brain responses and his clinical progression over time. The patient's pattern of brain responses to a speech-processing task had a remarkable correspondence with his residual awareness, as assessed using a standard bedside clinical examination. Specifically, the patient showed a selective recruitment of the left angular gyrus while listening to a native speech narrative (as compared to the reverse presentation of the same narrative) only when the patient's behavioral responses were consistent with the clinical criteria of an MCS. In addition, the patient showed an overall increase in BOLD signal in the language-related network and 
(a)

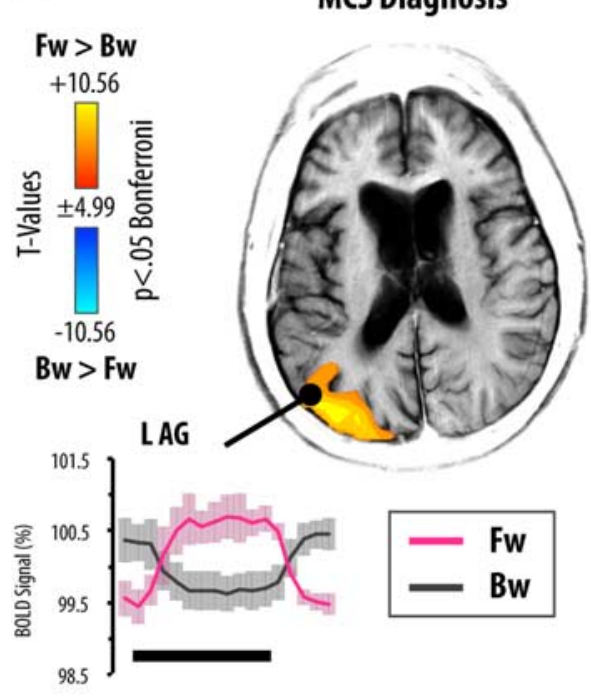

(b)

\section{Language Network $\square$ DMN $\square$ Visual Motion Perception (Control ROI)}

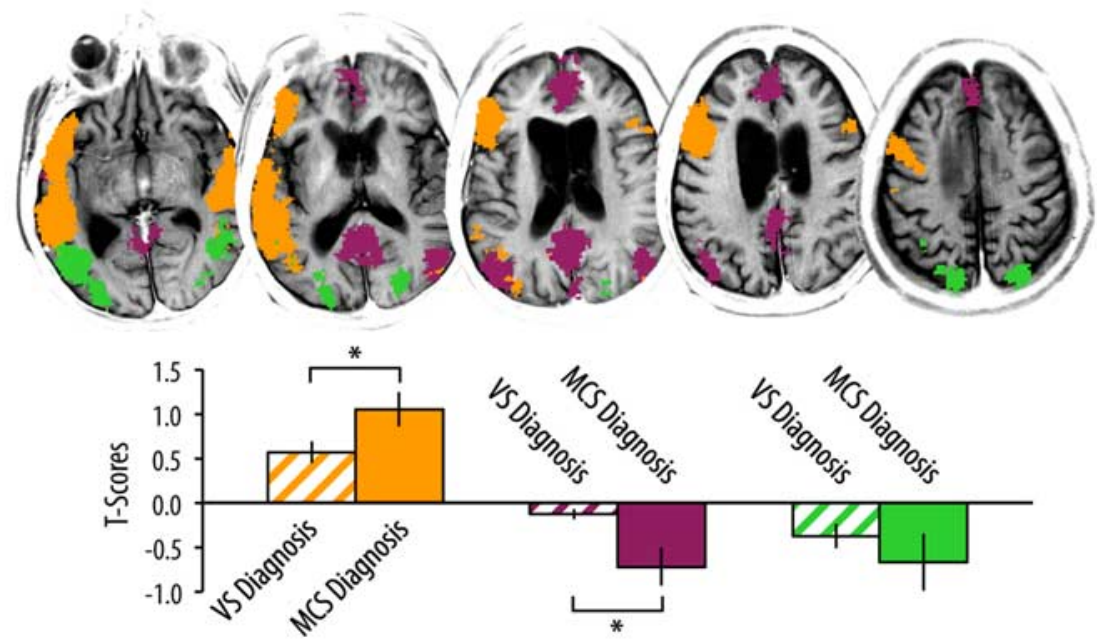

Fig. 2. Panel A depicts the results for the direct comparison of brain activity related to forward versus backward speech ( $p<.05$ Bonferroni corrected), after the patient had progressed to an MCS. The left angular gyrus (L AG) was selectively recruited during the presentation of natural native language narrative (red-yellow cluster; Fw $>\mathrm{Bw}$ ), as compared to the reverse speech. No significant brain regions were revealed by the opposite contrast $(\mathrm{Bw}>\mathrm{Fw})$. Panel $\mathrm{B}$ shows the effects of the patient clinical evolution toward consciousness in three meta-analytically defined brain networks: the first ROI comprises brain regions involved in language processing (i.e., language network; orange), the second represents the DMN (purple), and the third includes brain regions related to visual motion perception (i.e., non-task-related control network; green). As shown in the lower part of panel B, we found a significant increase of the hemodynamic activity (i.e., $t$ scores for both forward and backward conditions) for the whole language network $(p=.049)$ and a parallel signal decrease for DMN regions $(p=.022$ ), as the patient progressed from a VS (dashed columns) to an MCS (plain columns). Brain activity for the motion perception network (i.e., control ROI) did not vary longitudinally $(p=.454)$.

a greater deactivation in DMN regions as he progressed from a VS to an MCS. Overall, these findings suggest that a longitudinal brain functional assessment, even in a single patient with a DOC, can add valuable information to a bedside clinical examination.

In fact, from a clinical perspective, the accurate evaluation of residual consciousness in DOC patients represents a major challenge and misdiagnosis can reach worrisome rates. In addition, it has been demonstrated that behavior and consciousness can be dissociated in some patients (please see Owen et al., 2006 and Monti et al., 2010). When such uncertainty is evident, the use of an independent estimator may lead to a synergistic effect and improve the reliability of the diagnostic process. For instance, we can imagine a two-by-two response model for behavioral and neuroimaging-based assessments of consciousness. The possible outcomes of this model are: [a] responsive behavior or [b] unresponsive behavior for the bedside assessment, and [a] responsive brain activity or [b] unresponsive brain activity for the neuroimaging assessment. It is clear that, when the two independent measurements give the same results (i.e., responsive behavior and brain activity; unresponsive behavior and brain activity), the probability of both type I (false positive) and type II (false negative) errors are diminished significantly.

Contrary to what one would expect, however, the diagnostic process can also benefit when the outcomes are conflicting. Indeed, discrepancies between behavior and brain responses can encourage the clinician to reconsider the validity of both measures and foster test-retest reliability of neuroimaging data. Although diagnostic guidelines (Kalmar \& Giacino, 2005) indicate the stability through time as a relevant point for the behavioral assessment of DOC patients, most findings from the neuroimaging literature rely on a single evaluation of brain activity (see for instance Coleman et al., 2007 or the more recent Stender et al., 2014).

More importantly, to corroborate the clinical evolution, responsive brain activity from the neuroimaging assessment should be obtained from the single patient (as in our case), and this can be achieved only when the multidimensional assessment (e.g., brain and behavior) is carried out longitudinally, before and after the improvement of clinical status. Importantly, the neural responses of interest in this work were detected using a 1.5T MRI scanner that is widely available in standard clinical settings, suggesting that our approach could be incorporated into clinical practice with relative ease. However, additional experimental data should be collected to confirm the validity and reliability of our experimental paradigm.

\section{Significance of Changes in Brain Activity Related to Clinical Progression}

Our most noteworthy finding regarding our patient's neural responses during his clinical progression from a VS to an 
MCS is that the patient selectively recruited brain regions that are critical for language comprehension only while he was in an MCS. First, when the patient was in an MCS, but not a VS, he recruited the left angular gyrus when he listened to the native speech narrative $(\mathrm{FW}>\mathrm{Bw})$. This neural marker of native speech processing has an early ontogenetic development (Dehaene-Lambertz et al., 2002) and is maintained during adulthood (Perani et al., 1996).

The left angular gyrus is also involved in lexical storage in adults, implicated in the analysis of speech prosodic patterns used by newborns to identify their native language, and has an important role in auditory short-term verbal memory and auditory rehearsal (Dronkers, Wilkins, Van Valin, Redfern, $\&$ Jaeger, 2004). Second, the patient engaged the left inferior frontal gyrus when listening to the speech narrative only while he was in an MCS. Importantly, the left inferior frontal gyrus is involved in speech production and is recruited by healthy volunteers during passive listening (Pulvermuller, Shtyrov, \& Ilmoniemi, 2003; Wilson, Saygin, Sereno, \& Iacoboni, 2004). Lastly, the BOLD signal within the patient's language network increased after he transitioned from a VS to an MCS, which likely reflects his more sophisticated processing of the speech stimuli once he entered an MCS.

In summary, our patient's clinical progression from a VS to an MCS involved the specific recruitment of key brain regions for native speech processing. Together with the evidence that the patient produced motor acts reliably in response to simple verbal commands after he recovered awareness, we hypothesize that his regained ability to recognize, properly segment, and comprehend spoken language (at least for simple commands) is associated with specific neural markers of language processing.

A secondary finding that differentiated our patient's clinical progression from a VS to an MCS was a greater deactivation in his DMN during speech perception (Rest > $\mathrm{Fw}+\mathrm{Bw}$ ). In healthy volunteers, important nodes of the DMN show a signal increase during self-referential mental activities, and a signal reduction during externally focused attention tasks, as compared to rest (Gusnard \& Raichle, 2001). The magnitude of these deactivations increases with the attentive load of the task and is associated with the magnitude of the activation in task-relevant brain regions (McKiernan, Kaufman, Kucera-Thompson, \& Binder, 2003). The DMN activity of patients with DOC demonstrated a noteworthy overlap with CRS-R scores in a cross-sectional study (Vanhaudenhuyse et al., 2010) and appears crucial for sustained attention after traumatic brain injury (Bonnelle et al., 2011). Accordingly, we propose that the DMN deactivations we observed in our patient reflect his mental effort to focus on the linguistic stimuli. Critically, this neural marker of increased mental exertion was evident only when the patient regained awareness (i.e., progressed from a VS to an MCS).

When evaluating the patient's lateralized brain response elicited by both speech conditions versus the silent rest period $(\mathrm{Fw}+\mathrm{Bw}>\mathrm{Rest})$, we found that the BOLD signal peaked in the left temporal perisylvian cortex exclusively when he was in an MCS. These regions of the language-dominant hemisphere are a node for low-level perception of auditory information and are also involved in language processing. Nevertheless, lateralization of BOLD activity per se is not sufficient as a marker of awareness or language comprehension.

\section{The Contribution of a Longitudinal, Passive, Task- Related fMRI Protocol for Patients with DOC}

Previous cross-sectional studies involving language processing in patients with DOC identified a partial overlap between clinical diagnosis and task-related brain activity (Coleman et al., 2007). Hence, previous investigators have concluded that both patients in a VS and patients in an MCS show similar neural markers of speech processing. Aside from the high clinical misdiagnosis rate (Schnakers et al., 2009), the discrepancy between neuroimaging findings and behavioral signs of awareness may result from individual etiopathological (e.g., anoxic encephalopathy vs. traumatic brain injury), demographic (e.g., younger vs. older patients), and medical potentially confounding variables (e.g., relevant comorbidity, duration of coma after brain injury, etc.). All of these afore-mentioned variables are usually not evaluated in cross-sectional designs and may, therefore, influence between-group contrasts (e.g., VS . MCS).

Presumably for the same reason, neuroimaging studies often fail to offer valuable diagnostic and prognostic information in individual patients with DOC, even though the correspondence between the patterns of neural responses and clinical diagnosis appears adequate at the group level (Giacino et al., 2014). To combat this issue, investigators have called for a multimodal, longitudinal evaluation of neuroimaging-based assessments for patients with DOC (Giacino et al., 2014). Our current work addresses this request directly.

Importantly, the within-subject design of our work reduces the influence of certain confounds of cross-sectional cohort studies: factors that depend on individual clinical history and demonstrate prognostic value, such as etiology (Bernat, 2006), are time-invariant. Although, these factors may still affect the overall probability of recovery, they are unlikely to influence the relationship between neuroimaging findings and behavior across time. Thus, our work addresses an important short-coming of past cross-sectional investigations, with the added benefit of reducing some error that is inherent to between-subject designs.

Finally, when the multimodal evaluation of a patient is carried out in a double-blind manner (as it should be), measurement errors (i.e., misdiagnosis) can occur in both cross-sectional and longitudinal designs. However, a unique feature of a longitudinal investigation is that a patient's brain responses and behavior can be compared across time, which should reduce the overall misdiagnosis rate.

Resting state fMRI studies (see review Hannawi et al., 2015) and passive task-related paradigms (Bekinschtein et al., 2004; Boly et al., 2008; Coleman et al., 2007; 
Di et al., 2007; Schiff et al., 2005) have similar advantages and pitfalls regarding diagnosis and prognosis for patients with DOC (Giacino et al., 2014). Nonetheless, to monitor functional brain responses throughout the consciousness continuum, simple passive speech perception tasks may be more informative. In fact, the use of a block-design protocol together with a passive task ensures that brain activity related to stimulus processing, whether conscious or not, is sustained for a prolonged period (e.g., please refer to the BOLD time-course of Figures 1 and 2).

This approach also yields higher statistical power as compared to event-related designs (Buxton, Wong, \& Frank, 1998), while maintaining a shorter scan time. Furthermore, a recent study (Fernandez-Espejo, Norton, \& Owen, 2014) demonstrated that block-design paradigms could be used to assess brain activity reliably, even with the "lower-end" equipment typically available in clinical settings (i.e., $1.5 \mathrm{~T}$ vs. 3T MR scanners). Altogether, then, our passive speech perception task coupled with a block-design protocol provides a pragmatic and informative addition to clinical bedside examinations for patients with DOC.

\section{Limitations of the Study}

Although we have presented a strong correspondence between the behavioral assessment of awareness and task-related brain responses from an individual patient with a DOC, we ought to consider that neuroimaging evidence per se does not represent a direct measure of consciousness. This limitation is related intrinsically to the passive paradigm that has been adopted, since only active tasks (i.e., those tasks that assess command-following) may provide a marker of patient volition.

A third fMRI acquisition performed concomitantly to the patient's transition from MCS to severe disability would have added important information about the reliability of our experimental paradigm in measuring the correspondence between consciousness and brain activity. Nevertheless, as reported in the Methods section and in accordance with the Ethical Committee protocol, fMRI acquisitions could be performed exclusively as a complement to the clinical routine MRI exam. In fact, the patient did not undergo any other clinical MRI exam after hospital discharge.

The study of DOC by means of task-related brain activity requires adapting the passive stimulation paradigm to the individual patient. One must consider the location of any brain lesion that the patient may have and use the patient's residual perceptive abilities. Our passive listening task requires the preservation of the auditory pathways from the brainstem to the primary auditory cortex and associative regions in the language-dominant hemisphere. Hence, even though our aim is to adopt this experimental paradigm to study a larger cohort of patients with DOC longitudinally, we are aware that some patients will not be eligible due to lesions in specific task-related brain areas.

In conclusion, the results of the present case study indicate that longitudinal assessments of brain responses to passive stimuli can contribute to the definition of the clinical status in individual patients with DOC. These assessments represent an adequate counterpart of the bedside evaluation during the diagnostic decision making process. In addition, the use of a standard and widely available 1.5T MRI scanner, together with the ease of a passive auditory block-design paradigm, suggests that our approach could be incorporated effectively into clinical practice.

\section{ACKNOWLEDGMENTS}

This study was approved by the Ethical Committee at the University of Pisa, Italy. There are no conflicts of interest to be disclosed. F.T. and L.C. thank A.TRA.C.TO onlus for the continuous support and motivation. We thank Dr. Giacomo Handjaras for valuable suggestions. This work was supported by the Italian Minister of Health (E.R. and L.C., Young Researcher Project, Call 2011-2012, GR-2011-02347383). Drs. Tomaiuolo and Cecchetti contributed equally to this work.

\section{REFERENCES}

Bekinschtein, T., Leiguarda, R., Armony, J., Owen, A., Carpintiero, S., Niklison, J., ... Manes, F. (2004). Emotion processing in the minimally conscious state. Journal of Neurology, Neurosurgery, and Psychiatry, 75(5), 788.

Bekinschtein, T., Tiberti, C., Niklison, J., Tamashiro, M., Ron, M., Carpintiero, S., ... Manes, F. (2005). Assessing level of consciousness and cognitive changes from vegetative state to full recovery. Neuropsychological Rehabilitation, 15(3-4), 307-322. doi:10.1080/09602010443000443

Bernat, J.L. (2006). Chronic disorders of consciousness. Lancet, 367(9517), 1181-1192. doi:10.1016/S0140-6736(06)68508-5

Boly, M., Faymonville, M.E., Schnakers, C., Peigneux, P., Lambermont, B., Phillips, C., ... Laureys, S. (2008). Perception of pain in the minimally conscious state with PET activation: An observational study. Lancet Neurology, 7(11), 1013-1020. doi:10.1016/S1474-4422(08)70219-9

Boly, M., Tshibanda, L., Vanhaudenhuyse, A., Noirhomme, Q., Schnakers, C., Ledoux, D., ... Laureys, S. (2009). Functional connectivity in the default network during resting state is preserved in a vegetative but not in a brain dead patient. Human Brain Mapping, 30(8), 2393-2400. doi:10.1002/ hbm. 20672

Bonnelle, V., Leech, R., Kinnunen, K.M., Ham, T.E., Beckmann, C.F., De Boissezon, X., ... Sharp, D.J. (2011). Default mode network connectivity predicts sustained attention deficits after traumatic brain injury. The Journal of Neuroscience, 31(38), 13442-13451. doi:10.1523/JNEUROSCI.1163-11.2011

Buxton, R.B., Wong, E.C., \& Frank, L.R. (1998). Dynamics of blood flow and oxygenation changes during brain activation: The balloon model. Magnetic Resonance in Medicine, 39(6), 855-864.

Caplan, R., \& Dapretto, M. (2001). Making sense during conversation: An fMRI study. Neuroreport, 12(16), 3625-3632.

Coleman, M.R., Rodd, J.M., Davis, M.H., Johnsrude, I.S., Menon, D.K., Pickard, J.D., \& Owen, A.M. (2007). Do vegetative patients retain aspects of language comprehension? Evidence from fMRI. Brain, 130(Pt 10), 2494-2507. doi:10.1093/brain/ awm 170 
Cox, R.W. (1996). AFNI: Software for analysis and visualization of functional magnetic resonance neuroimages. Computers and Biomedical Research, 29(3), 162-173.

Dehaene-Lambertz, G., Dehaene, S., \& Hertz-Pannier, L. (2002). Functional neuroimaging of speech perception in infants. Science, 298(5600), 2013-2015. doi:10.1126/science.1077066

Demertzi, A., Gomez, F., Crone, J.S., Vanhaudenhuyse, A., Tshibanda, L., Noirhomme, Q., ... Soddu, A. (2014). Multiple fMRI system-level baseline connectivity is disrupted in patients with consciousness alterations. Cortex, 52, 35-46. doi:10.1016/ j.cortex.2013.11.005

Di, H.B., Yu, S.M., Weng, X.C., Laureys, S., Yu, D., Li, J.Q., ... Chen, Y.Z. (2007). Cerebral response to patient's own name in the vegetative and minimally conscious states. Neurology, 68(12), 895-899. doi:10.1212/01.wnl.0000258544.79024.d0

Dronkers, N.F., Wilkins, D.P., Van Valin, R.D., Jr., Redfern, B.B., \& Jaeger, J.J. (2004). Lesion analysis of the brain areas involved in language comprehension. Cognition, 92(1-2), 145-177. doi:10.1016/j.cognition.2003.11.002

Fernandez-Espejo, D., Norton, L., \& Owen, A.M. (2014). The clinical utility of fMRI for identifying covert awareness in the vegetative state: A comparison of sensitivity between 3T and 1.5T. PLoS One, 9(4), e95082. doi:10.1371/journal.pone.0095082

Fernandez-Espejo, D., \& Owen, A.M. (2013). Detecting awareness after severe brain injury. Nature Reviews Neuroscience, 14(11), 801-809. doi:10.1038/nrn3608

Giacino, J.T., Ashwal, S., Childs, N., Cranford, R., Jennett, B., Katz, D.I., ... Zasler, N.D. (2002). The minimally conscious state definition and diagnostic criteria. Neurology, 58(3), 349-353. doi:10.1212/WNL.58.3.349

Giacino, J.T., Fins, J.J., Laureys, S., \& Schiff, N.D. (2014). Disorders of consciousness after acquired brain injury: The state of the science. Nature Reviews Neurology, 10(2), 99-114. doi:10.1038/nrneurol.2013.279

Giacino, J.T., \& Smart, C.M. (2007). Recent advances in behavioral assessment of individuals with disorders of consciousness. Current Opinion in Neurology, 20(6), 614-619. doi:10.1097/ WCO.0b013e3282f189ef

Gusnard, D.A., \& Raichle, M.E. (2001). Searching for a baseline: Functional imaging and the resting human brain. Nature Reviews Neuroscience, 2(10), 685-694. doi:10.1038/35094500

Hannawi, Y., Lindquist, M.A., Caffo, B.S., Sair, H.I., \& Stevens, R.D. (2015). Resting brain activity in disorders of consciousness: A systematic review and meta-analysis. Neurology, 84(12), 1272-1280. doi:10.1212/WNL.0000000000001404

Jenkinson, M., Beckmann, C.F., Behrens, T.E., Woolrich, M.W., \& Smith, S.M. (2012). Fsl. Neuroimage, 62(2), 782-790. doi:10.1016/j.neuroimage.2011.09.015

Kalmar, K., \& Giacino, J.T. (2005). The JFK Coma Recovery Scale-Revised. Neuropsychological Rehabilitation, 15(3-4), 454-460. doi:10.1080/09602010443000425

Laureys, S., Lemaire, C., Maquet, P., Phillips, C., \& Franck, G. (1999). Cerebral metabolism during vegetative state and after recovery to consciousness. Journal of Neurology, Neurosurgery, and Psychiatry, 67(1), 121.

Laureys, S., Owen, A.M., \& Schiff, N.D. (2004). Brain function in coma, vegetative state, and related disorders. Lancet Neurology, 3(9), 537-546. doi:10.1016/S1474-4422(04)00852-X

Lutkenhoff, E.S., Rosenberg, M., Chiang, J., Zhang, K., Pickard, J.D., Owen, A.M., \& Monti, M.M. (2014). Optimized brain extraction for pathological brains (optiBET). PLoS One, 9(12), e115551. doi:10.1371/journal.pone.0115551
McKiernan, K.A., Kaufman, J.N., Kucera-Thompson, J., \& Binder, J.R. (2003). A parametric manipulation of factors affecting task-induced deactivation in functional neuroimaging. Journal of Cognitive Neuroscience, 15(3), 394-408. doi:10.1162/089892903321593117

Monti, M.M., Pickard, J.D., \& Owen, A.M. (2013). Visual cognition in disorders of consciousness: From V1 to top-down attention. Human Brain Mapping, 34(6), 1245-1253. doi:10.1002/ hbm. 21507

Monti, M.M., Vanhaudenhuyse, A., Coleman, M.R., Boly, M., Pickard, J.D., Tshibanda, L., ... Laureys, S. (2010). Willful modulation of brain activity in disorders of consciousness. New England Journal of Medicine, 362(7), 579-589. doi:10.1056/ NEJMoa0905370

Multi-Society Task Force on PVS. (1994). Medical Aspects of the Persistent Vegetative State. New England Journal of Medicine, 330(21), 1499-1508. doi:10.1056/NEJM199405263302107

Naci, L., \& Owen, A.M. (2013). Making every word count for nonresponsive patients. JAMA Neurology, 70(10), 1235-1241. doi:10.1001/jamaneurol.2013.3686

Owen, A.M., Coleman, M.R., Boly, M., Davis, M.H., Laureys, S., \& Pickard, J.D. (2006). Detecting awareness in the vegetative state. Science, 313(5792), 1402. doi:10.1126/science.1130197

Perani, D., Dehaene, S., Grassi, F., Cohen, L., Cappa, S.F., Dupoux, E., ... Mehler, J. (1996). Brain processing of native and foreign languages. Neuroreport, 7(15-17), 2439-2444.

Poldrack, R.A., Mumford, J.A., Schonberg, T., Kalar, D., Barman, B., \& Yarkoni, T. (2012). Discovering relations between mind, brain, and mental disorders using topic mapping. PLoS Computational Biology, 8(10), e1002707. doi:10.1371/journal.pcbi.1002707

Pulvermuller, F., Shtyrov, Y., \& Ilmoniemi, R. (2003). Spatiotemporal dynamics of neural language processing: An MEG study using minimum-norm current estimates. Neuroimage, 20(2), 1020-1025. doi:10.1016/S1053-8119(03)00356-2

Schiff, N.D., Rodriguez-Moreno, D., Kamal, A., Kim, K.H., Giacino, J.T., Plum, F., \& Hirsch, J. (2005). fMRI reveals large-scale network activation in minimally conscious patients. Neurology, 64(3), 514-523. doi:10.1212/01. WNL.0000150883.10285.44

Schnakers, C., Vanhaudenhuyse, A., Giacino, J., Ventura, M., Boly, M., Majerus, S., ... Laureys, S. (2009). Diagnostic accuracy of the vegetative and minimally conscious state: Clinical consensus versus standardized neurobehavioral assessment. BMC Neurology, 9, 35. doi:10.1186/1471-2377-9-35

Stender, J., Gosseries, O., Bruno, M.A., Charland-Verville, V., Vanhaudenhuyse, A., Demertzi, A., ... Laureys, S. (2014). Diagnostic precision of PET imaging and functional MRI in disorders of consciousness: A clinical validation study. Lancet, 384(9942), 514-522. doi:10.1016/S0140-6736(14) 60042-8

Teasdale, G., \& Jennett, B. (1974). Assessment of coma and impaired consciousness. A practical scale. Lancet, 2(7872), $81-84$.

Vanhaudenhuyse, A., Noirhomme, Q., Tshibanda, L.J., Bruno, M.A., Boveroux, P., Schnakers, C., ... Boly, M. (2010). Default network connectivity reflects the level of consciousness in non-communicative brain-damaged patients. Brain, 133(Pt 1), 161-171. doi:10.1093/brain/awp313

Wilson, S.M., Saygin, A.P., Sereno, M.I., \& Iacoboni, M. (2004). Listening to speech activates motor areas involved in speech production. Nature Neuroscience, 7(7), 701-702. doi:10.1038/nn1263 
Yarkoni, T., Poldrack, R.A., Nichols, T.E., Van Essen, D.C., \& Wager, T.D. (2011). Large-scale automated synthesis of human functional neuroimaging data. Nature Methods, 8(8), 665-670. doi:10.1038/nmeth.1635

\section{APPENDIX}

Original Italian Version of the Short Narrative (Forward Speech Stimulus)

Il sole rischiarava le ombre che il tramonto già proiettava sul giardino. La luce tenue filtrava lungo il corridoio e lasciava una scia brillante, che contrastava con lo scuro delle pareti circostanti. La mia stanza dava sul giardino e l'unica finestra, con le tende tirate sui lati, dava l'impressione di far entrare la grande quercia.

English Version of the Short Narrative (Forward Speech Stimulus)

The sunset was already casting shadows onto the garden, although the sun was still dimly filtering through the hallway in a blade of bright light contrasting with the dark walls. My room had a garden view, and the only window, its curtains pulled to the sides, almost seemed to be inviting the large oak tree in. 